Mrs. Birgitta Dahl-Kettner. Dr. Widstrand is also executive secretary of the Swedish Nubia Committee.

Seminars and courses already held in 1964 include a seminar on the development of adult education in Africa (14-16 October), and courses for experts engaged in the Scandinavian Tanganyika Project (II-17 October) and on problems of African development (14-is November). Others are planned for the future. The first number of the Institute's own series of publications in Swedish came out in June 1964. The Institute is now in close touch with other scientific libraries in Scandinavia and further agreements have been reached on the supply of catalogue cards for the Institute library's joint catalogue.

\title{
Etude des populations de pêcheurs du Golfe de Guinée
}

LE peuplement des zones méridionales du Togo, du Ghana et de la Côte d'Ivoire s'est principalement effectué - et continue de s'effectuer par le biais des migrations de la maind'œuvre - suivant un axe nord-sud ou nord-est-sud-ouest. Mais la côte du Golfe de Guinée a constitué de son côté - et continue de constituer de la même manière - une voie de déplacements de populations essentiellement adonnées à la pêche. Nous pouvons rencontrer aujourd'hui, notamment, des 'Fanti' et des Ewe disséminés, depuis leur pays d'origine, jusqu'à Conakry et jusqu'à Pointe Noire.

L'étude des principales populations de pêcheurs qui opèrent le long de cette côte - de leurs migrations historiques et saisonnières; des relations et des échanges qu'elles ont entretenus ou entretiennent entre elles et avec les groupes des rivages d'accueil; des aspects originaux de leur organisation économique et sociale ainsi que de leurs traditions religieuses; des problèmes socio-économiques, enfin, auxquelles elles se trouvent confrontées en raison du progrès technique - constitue le sujet des travaux en question.

Il s'agit de travaux à long terme qui ont débuté par une enquête réalisée d'Octobre 1963 à Mars 1964 auprès des pêcheurs de Côte d'Ivoire.

Cette enquête a donné lieu à un rapport ronéotypé dont le tome I traite des pêcheurs maritimes (1. Les pêcheurs Aulo et assimilés; 2. les pêcheurs Fanti; 3. les pêcheurs Alladian, Nanakrou et divers). Le tome II traite des pêcheurs lagunaires (N'zina, Essouman, Ehoutilé, Adioukrou, Ahizi, Avikam, Dida, Sénégalais et Maliens).

Le rapport dresse une sorte de tableau de la pêche africaine de type traditionnel en Côte d'Ivoire; il précise l'effectif, la localisation et les techniques de pêche des différents groupes de pêcheurs, et fait apparaître les motifs, les modalités et l'intensité des migrations saisonnières de la plupart d'entre eux (la totalité des pêcheurs maritimes professionnels). Il s'attache principalement à analyser les structures, les conditions de création et les lois de fonctionnement des diverses unités de production, notamment des ' compagnies de pêche' dont les effectifs varient, selon les cas, de 5 à 80 personnes. Il évoque à cette occasion les principaux problèmes économiques de la pêche traditionnelle. Il contient, enfin, une série de notes sommaires sur la vie sociale et religieuse des populations visitées.

L'étude se poursuivra en 1965 par une enquête qui sera menée, cette fois, dans les régions d'origine des pêcheurs 'Fanti' et Ewe. Elle mettra en lumière à quel point le phénomène des migrations, en faisant varier sur une large échelle - comme nulle expérience n'aurait ambitionné de la faire - aussi bien la taille que l'environnement ou le degré d'isolement des campements de pêcheurs, constitue une voie d'investigation féconde.

[Communication de M. Albert de Surgy, attaché de recherches au Centre National de la Recherche Scientifique, Paris]

\section{University of Ghana, Institute of African Studies: 'Research Review'}

THE first number of the Research Review of the Institute of African Studies at the University of Ghana appeared in the Lent Term 1965, under the editorship of Mr. Kwame Arhin, 